\title{
Outpatient Termination of Pregnancy
}

\author{
STELLA C. LEWIS， SHIRLEY LAL， B. BRANCH， R. W. BEARD
}

British Medical fournal, 1971, 4, 606-610

\section{Summary}

Termination of pregnancies ranging from 6 to 10 weeks' gestation is described in 127 women who attended the hospital as outpatients. The technique employed was to aspirate the products of conception through a narrow plastic tube using a high negative pressure. Infiltration of the cervix with local anaesthetic proved so effective that the procedure could be carried out on the fully conscious patient. The short time taken to evacuate the uterus, the small blood loss, and the low incidence of complications in the latter part of the study suggest that the technique is a valuable procedure. Relatively more women can be terminated as outpatients than as inpatients with a corresponding reduction in the demands made on gynaecological beds.

\section{Introduction}

Until 1967 legal abortion presented no problem to the average hospital in Britain. Then with the advent of the Abortion Act the number of patients requesting termination of pregnancy increased considerably, and in the full year of 197043,224 abortions were performed under the National Health Service. ${ }^{1}$ Most of these women were admitted into beds which until then had apparently been fully utilized by gynaecological patients.

At King's College Hospital referrals for termination are accepted only from the hospital district, which has a population of about 300,000. During 1970794 terminations were performed. ${ }^{2}$ The patients were accommodated within the existing facility of the gynaecological department by the more efficient use of beds and by the earlier discharge of patients. A national survey showed a similar trend. ${ }^{3}$ Nevertheless, it was clear that if the admission of these patients continued the existing facilities would become inadequate. For this reason it was decided to investigate the possibilities of the so-called "lunchtime" abortion procedures reported from such centres as the Women's Clinic in New York. ${ }^{4}$

This study reports the results in the first 133 patients who had outpatient terminations performed by the Karman catheter aspiration technique.

\section{Patients and Methods}

The study extended over a five-month period from 1 February to 25 June 1971. During this time outpatient terminations were carried out on 133 patients. In order to show trends developing during the course of the study the patients are divided into three groups. Six patients were not included in the study - two had missed abortions and four were more than 11

\footnotetext{
Department of Obstetrics and Gynaecology, King's College Hospital, London S.E.5

STELLA C. LEWIS, M.B., B.S., M.R.C.o.G., Research Registrar

SHIRLEY LAL, B.A., A.I.M.s.W., Research Social Worker

R. W. BEARD, M.D., M.R.C.o.G., Senior Lecturer

Preterm Inc., Washington D C.

B. BRANCH, M.D., Medical Director
}

weeks pregnant (from date of the last menstrual period). Thus the results were obtained from 127 patients-33 in group 1, 46 in group 2, and 48 in group 3 . The ages of the patients ranged from 16 to 45 years. Altogether $38 \%$ were single, $52 \%$ were married, and $10 \%$ were divorced or separated. Forty-one patients were nulliparae and 86 were multiparae, of whom 13 had had five or more pregnancies. Ten women had had a previous legal abortion.

\section{ADMINISTRATION}

To see as many patients as possible before they were more than 10 weeks pregnant a clinic appointment was given for a day not more than one week after the referral letter from their general practitioner was received. During the period of study $43 \%$ of the patients were of 10 weeks' gestation or less, and $73 \%$ of all referrals were accepted for termination. After acceptance the patient was interviewed by a social worker as part of a larger study devoted to the physical and psychosocial aspects of legal abortion. At the same time the termination procedure was explained to the patient and she was given the option of having a general anaesthetic, which four patients accepted. After acceptance for termination an explanatory leaflet was given to every patient (see Important notice).

\section{IMPORTANT - PLEA SE READ CAREFULLY}

You have requested termination of your pregnancy at an early stage. Because of this it will be possible to perform the procedure on an outpatient basis. The method is simple but your cooperation and confidence in the doctor are important if it is to be successful.

When you come to the hospital you will be shown into a small sitting room. After a short discussion with a member of the staff, when you will be able to ask any questions, you will be taken to the examination room, and after getting onto a low bed you will be given a gentle internal examination by the doctor. In order to carry out the termination a plastic tube is passed through the neck of the womb, but it is unusual for you to feel this part of the procedure. You will then hear the sound of a suction machine which empties the womb. The suction may produce a cramp-like discomfort similar to having a normal period but many women have no discomfort at all. The whole procedure takes 5 to 10 minutes. Afterwards you will be able to lie down and you should be ready to go home within $2-3$ hours. Coffee or a light lunch is provided.

If you have any anxieties before the termination please do not hesitate to discuss them with the doctor.

The interview and leaflet were found to be important preliminaries to outpatient termination for obtaining the confidence and subsequent co-operation of the patient. Contraception was also discussed fully so that the patient was acquainted with the various techniques available when she attended for follow-up. All terminations were carried out within one week of the initial visit to the hospital. They were performed in a small outpatient theatre adjacent to a 14-bedded short-stay ward which is used for a variety of gynaecological procedures, one half-day a week being devoted to outpatient terminations. On the day of operation patients arrived between 8.15 and 8.30 a.m. The number of abortions performed in one morning session ranged from 4 to 13. Patients were allowed to go home after an early lunch. Those with a slight rise in temperature, abdominal pain, or bleeding were detained overnight. Before discharge every 
patient was asked to contact the duty gynaecological house surgeon if she had any anxieties before her next appointment. At the same time a letter was sent to the patient's general practitioner (so that it would arrive by the following day) informing him of the procedure. Appointments for follow-up clinics 10 days, six weeks, and three months after the termination were given to every patient. The six-week clinic was primarily contraceptive in purpose.

\section{ANALGESIA}

Patients were fully conscious throughout the procedure and no form of oral or intravenous premedication was administered. Initially no local anaesthetic was given for the procedure (33 patients) but subsequently paracervical block (46 patients) was used, and in the last part of the study intracervical block (48 patients). The technique of this last procedure was to inject $12 \mathrm{ml}$ of $1 \%$ lignocaine (Xylocaine) into the substance of the cervix at the 12, 4, and 8 o'clock positions. The analgesic effect of these different approaches are described below.

\section{KARMAN CATHETER}

The Karman catheter* is a flexible length of polyethylene tubing closed at one end with a rounded tip immediately below which are two eyes placed in series. The eyes are so placed that if the catheter is pushed against the vault of the uterine cavity the tip will collapse rather than perforate the uterus. The catheter and its mode of action when suction is applied are shown in the Diagram. The products of conception are sucked in through the eyes; large portions of placenta or embryo are either compressed or broken up as they enter the catheter lumen. The upper edge of each catheter eye is convex and acts as a curette as the uterine wall is sucked against the catheter.

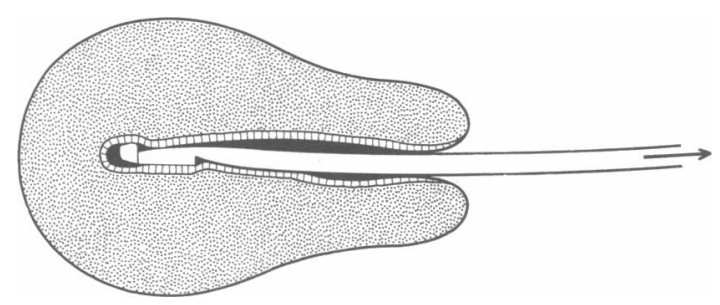

Diagrammatic representation of curetting action of the Karman catheter when suction is applied.

\section{TECHNIQUE}

The patient was accompanied to the theatre by the social worker who had previously interviewed her. Immediately before this she had emptied her bladder. She was placed in the lithotomy position and the size of the uterus was assessed by bimanual examination. After a cervical smear and high vaginal swab for bacteriological examination had been taken through a Cusco speculum the cervix and vagina were thoroughly cleaned with povidone-iodine. The vaginal mucosa covering the anterior cervix was seized transversely with a single-toothed tenaculum. The cervix or paracervical tissues were injected with local anaesthetic (apart from the early cases in which local anaesthetic was not used). The Karman catheter (6 mm diameter) was then introduced into the uterus, usually without dilatation of the cervix. In some nulliparae with a pinhole os it was necessary to dilate the cervix up to $7 \mathrm{~mm}$. The vault of the uterine cavity could be felt as soon as the catheter reached it. If no local anaesthetic was used the response of the patient was unpredictable, varying from minimal discomfort to pain, but in the anaesthetized groups the procedure was almost painless.

Once the catheter was in the uterus it was attached to rubber pressure tubing and a negative pressure of $26-29$ in $\mathrm{Hg}$ was applied by means of an electrical suction pump. $\dagger$ By gently moving the catheter up and down from the internal os to the fundus and rotating it at the same time the uterus was rapidly emptied. Passage of the products of conception could be seen without difficulty through the catheter wall, and if it stopped it usually signified that the catheter was blocked by a piece of placenta or membrane. Withdrawal of the catheter from the uterus and removal of tissue from the eye was enough to reestablish the flow. When the uterine cavity was almost empty a rough sensation, easily felt by the operator, was transmitted down the catheter as it was pulled down the wall of the uterus. When this sensation was obtained from all parts of the uterine cavity it usually signified that the abortion was complete. Shortly after the study began the practice of exploring the uterus with a small Friedman curette after suction was completed was found to be invaluable for ensuring that the cavity of the uterus was empty. No oxytocic was ever required to control bleeding as the uterus always contracted tightly during the procedure. As a final routine it became customary to examine the evacuated material suspended in water to ensure that genuine products of conception were present. The case history of a patient in whom this was not done is given below.

\section{MEASUREMENTS}

After the products of conception were examined they were sent to the pathologist to be inspected and weighed. The combined blood and amniotic fluid volume was measured. Time taken from the onset of suction to the complete evacuation of the uterus was also recorded. Suction time should be distinguished from the time taken for the whole procedure described by Strausz and Schulman. ${ }^{5}$

An attempt was made to assess the pain response and general reaction of patients to the procedure. Before going home patients were asked if they had experienced any pain and if so how severe it was. They were also asked whether they had found the operation "quite acceptable," "tolerable," or "unbearable" and if they would have preferred a general anaesthetic. At the sixweek follow-up clinic the questions were repeated.

\section{Results}

\section{SUCTION TIME}

The time taken to empty the uterus was accurately determined in 96 patients, and the mean values for the three groups of patients are shown in Table I. As the study progressed the suction time decreased, and there was a significant difference between the mean suction time of 3 minutes 28 seconds for group 1 and of 2 minutes 30 seconds for group $3(P<0 \cdot 01)$. The large standard deviations, however, showed that there was considerable variation in suction time in all the groups. There was a positive correlation between suction time and the crude weight of the products of conception, which was significant $(\mathrm{r}+0.51 ; \mathrm{P}<0.01)$. Accurate weighing of all solid material remaining after sieving was carried out on 119 specimens,

TABLE I-Mean suction time for patients in groups 1, 2, and 3. Difference between mean suction time for groups 1 and 3 is significant $(P<0 \cdot 01)$.

\begin{tabular}{|c|c|c|c|}
\hline & Group & No. of Patients & Mean Suction Time in Sec \pm S.D. \\
\hline $\begin{array}{l}1 \\
2 \\
3\end{array}$ & $\begin{array}{ll} & \end{array}$ & $\begin{array}{l}22 \\
32 \\
42\end{array}$ & $\begin{array}{l}208 \cdot 2 \pm 102 \cdot 3 \\
174 \cdot 3 \pm 65 \cdot 36 \\
151 \cdot 28 \pm 59 \cdot 77\end{array}$ \\
\hline
\end{tabular}

†Eschman Bros. and Walsh Ltd., Shoreham-by-Sea, Sussex. 
TABLE II-Crude net weight of evacuated products of conception related to duration of pregnancy (in weeks) in 119 patients.

\begin{tabular}{|c|c|c|c|}
\hline \multicolumn{2}{|c|}{ Period of Gestation } & No. of Patients & Mean Weight in $\mathbf{g} \pm$ S.D. \\
\hline $\begin{array}{l}<7 \\
7-7+6 \cdots \\
8-8+6 \\
9-9+6 \\
10-10+6\end{array}$ & $\begin{array}{ll}\ldots & \ldots \\
\ldots & \ldots \\
\ldots & \ldots \\
\ldots & \ldots\end{array}$ & $\begin{array}{r}2 \\
27 \\
32 \\
37 \\
21\end{array}$ & $\begin{array}{l}46 \cdot 0 \\
38 \cdot 4 \pm 14 \cdot 3 \\
48 \cdot 4 \pm 18 \cdot 1 \\
56 \cdot 8 \pm 15 \cdot 3 \\
67.9 \pm 23.4\end{array}$ \\
\hline
\end{tabular}

TABLE III-Mean estimated blood loss including amniotic fluid in 121 patients from groups 1,2, and 3. Difference between mean losses for groups 1 and 2 and 3 was highly significant $(P \quad 0.001)$.

\begin{tabular}{|c|c|c|c|}
\hline & Group & No. of Patients & Mean Blood Loss in $\mathrm{ml} \pm$ S.D. \\
\hline $\begin{array}{l}1 \\
2 \\
3\end{array}$ & $\begin{array}{ll}\ldots & \ldots \\
\ldots & \ldots \\
\ldots & \end{array}$ & $\begin{array}{l}37 \\
39 \\
45\end{array}$ & $\begin{array}{r}103.7 \pm 61.9 \\
62.3 \pm 23.3 \\
58.9 \pm 35.4\end{array}$ \\
\hline
\end{tabular}

TABLE IV-Mean blood loss including amniotic fluid related to week of pregnancy from last menstrual period.

\begin{tabular}{|c|c|c|c|}
\hline \multicolumn{2}{|c|}{ Period of Gestation } & No. of Patients & Mean Blood Loss in $\mathrm{ml} \pm$ S.D. \\
\hline $\begin{array}{l}<7 \\
7-7+6 \\
8-8+6 \\
9-9+6 \\
10-10+8\end{array}$ & $\begin{array}{ll}\ldots & \ldots \\
\ldots & \ldots \\
\ldots & \ldots \\
\cdots & \ldots\end{array}$ & $\begin{array}{r}2 \\
27 \\
33 \\
38 \\
21\end{array}$ & $\begin{array}{l}55.0 \\
55.6 \pm 31.7 \\
66.1 \pm 39.1 \\
85.7 \pm 46.5 \\
89.3 \pm 66.4\end{array}$ \\
\hline
\end{tabular}

TABLE $\mathrm{V}-$ General reaction and pain response among patients in the analgesia groups. Numbers of patients stating that they would have preferred a general groups. Numbers of patients stating
anaesthetic are shown in parentheses.

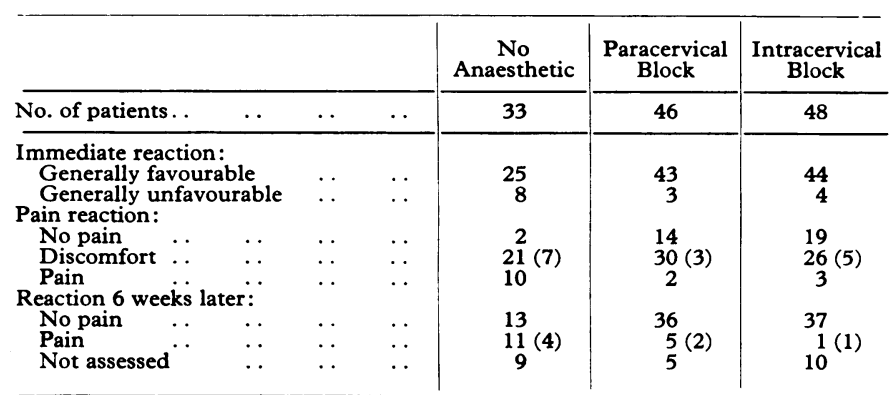

TABLE VI-Postoperative complications requiring readmission to hospital in groups 1,2 , and 3 .

\begin{tabular}{|c|c|c|c|c|}
\hline & Group & Excessive Bleeding & Pyrexia/Abdominal Pain & Other \\
\hline $\begin{array}{l}1 \\
2 \\
3\end{array}$ & $\begin{array}{ll} & \\
\cdots & \\
. & \end{array}$ & $\begin{array}{l}5 \\
3 \\
0\end{array}$ & $\begin{array}{l}2 \\
0 \\
1\end{array}$ & $\begin{array}{l}3 \\
2 \\
1\end{array}$ \\
\hline
\end{tabular}

and the results related to gestation are shown in Table II. As expected, the more prolonged the pregnancy the greater the weight of products, but the wide variations recorded between the weights of products from pregnancies of the same gestational period was surprising. As expected from these relationships there was also a positive correlation between the period of gestation and suction time.

\section{BLOOD LOSS (INCLUDING AMNIOTIC FLUID)}

Blood loss was measured accurately in 121 patients. The mean loss for each patient group is shown in Table III. As the study progressed there was a significant reduction in blood loss accompanying the shorter suction time. The wide variation in the mean loss shown for each patient group is partly accounted for by the influence of gestation. A definite trend of increasing blood loss with advancing pregnancy is shown in Table IV. No significant relation was found between blood loss and the parity of the patient.

\section{PAIN RESPONSE}

The responses received from the 127 patients to direct questions within three hours of the procedure and again six weeks later related to the analgesic technique used are shown in Table V. Regardless of analgesia the overall impression of the procedure was favourable among most of the patients $(89 \%)$. Of the 33 patients who were not given an analgesic 31 complained of either mild or moderate pain during the procedure when questioned. It is interesting to note, however, that six weeks later 13 of these patients did not remember the procedure as being painful.

A considerable reduction in the pain experienced is apparent in both the paracervical and intracervical block groups; out of 94 patients only 5 experienced any form of pain. The remainder experienced no pain at all or only discomfort. Both methods seem equally effective for the relief of pain from these results, though the impression of both operators (R.W.B. and S.C.L.) was that intracervical block was more effective than paracervical block. Despite the reduced pain response among patients in the local anaesthetic groups the number of patients who stated that they would have preferred a general anaesthetic, though small, remained about the same as in the group who did not have anaesthesia. The major reason given by most of these patients for this choice was not the discomfort of the procedure but their dislike of any form of participation in the actual termination.

\section{POSTOPERATIVE COMPLICATIONS}

One patient was detained in hospital postoperatively and 16 were subsequently admitted to hospital. The indications for admission are listed in Table VI. Clearly the incidence of postoperative complications declined sharply between groups 1 and 2 and a further decline occurred between groups 2 and 3. Eight patients were admitted with bleeding but none required transfusion. Retained products were present in five of these. Three patients had clinical evidence of acute pelvic sepsis and responded rapidly to antibiotic therapy. In no patient who developed the signs of acute pelvic sepsis was an organism grown from the high vaginal swab before operation or on readmission. Other complications requiring admission included urinary symptoms in three patients, though on culture the urine was sterile, one case of ectopic pregnancy (this case is described belcw), and a case of acute bronchitis. Finally, one patient was kept in after the termination because the tip of a catheter had snapped off in the uterine cavity. The tip was found without difficulty when the uterus was explored under general anaesthesia on the following day. This incident occurred at a time when it was necessary to re-use catheters and is not a hazard if they are used only once.

When patients attended the clinic on the 10th postoperative day they often complained of a few days' vaginal bleeding, occasionally accompanied by the loss of clots, starting two or three days after the operation. Bleeding heavier than normal menstruation was recorded in 24 out of 119 patients and resolved spontaneously without treatment. All patients were seen finally three months after the operation. No further complications had developed in any of them between the postoperative visits at 10 days and three months.

\section{CASE HISTORY}

A married woman was accepted for termination when she was nine weeks pregnant. She had no complaints and on vaginal 
examination the uterus was thought to be enlarged to the expected size for the gestational period. At operation $40.7 \mathrm{~g}$ of tissue was aspirated from the uterine cavity, which was within normal limits. Ten days later she was admitted with classical clinical signs of a ruptured ectopic pregnancy, which was confirmed at operation. Placental fragments can easily be identified by naked-eye examination if the aspirated material is suspended in water. It seems likely that if the products had been examined more closely and the absence of villi noted immediately after operation an earlier diagnosis of ectopic pregnancy might have been made.

\section{CONTRACEPTION}

Seventy-two patients (57\%) were not using any form of contraception when they became pregnant, while in the remaining $55(43 \%)$ the stated cause of pregnancy was a failure of some method of contraception. A total of 110 patients $(87 \%)$ attended the second follow-up clinic six weeks after their operation. Contraception was provided for all these patients (oral contraception for 81, and a Lippes loop for 22). In two patients a Lippes loop was inserted at the time of operation and seven patients were put on the waiting list for sterilization.

\section{Discussion}

The short suction time and small blood loss reported in this study show that by use of a high negative pressure the uterus can be safely and efficiently evacuated through a narrow tube. Though no comparable figures are available for other techniques probably the Karman catheter method is the most rapid available for pregnancies up to 10 weeks' gestation. The mechanical efficiency of this method is dependent on a high negative pressure, which has always been thought dangerous because of the risk of damaging the uterine wall. If the area of uterine wall exposed to the vacuum is critical, however, then the small eye of the Karman catheter permits the use of a much higher vacuum pressure than does, for example, the larger eye of the standard metal Bierer cannula.

Termination as an outpatient procedure though widely used in other countries ${ }^{6} 7$ is not commonly practised in Britain. In fact the Department of Health recommend that patients should be kept under observation overnight or longer. The rationale behind the recommendation is that termination carries a small but definite risk of severe postoperative haemorrhage from which the patient could die if she were not in hospital. Recently several well-documented reports of termination on many women have shown that the risk is negligible for terminations done in the first trimester of pregnancy. ${ }^{5}{ }^{7}$ Potts and Branch ${ }^{6}$ commented that clinical experience had shown that most complications arise either at the time of operation or three to seven days afterwards, and we agree with this view. Thus the argument in favour of a period of stay in hospital after operation for women having a first trimester termination is less valid.

This study reports the initial experience followed by the gradual improvement in results commonly obtained when a new technique is introduced into clinical practice. Of particular concern among the patients early in the study (group 1) was the high incidence of postoperative complications (see Table V). Incomplete evacuation of the uterus was probably the major cause of postoperative sepsis and bleeding, and it was soon clear that the inexperienced operator at least could not be certain with the Karman catheter alone that the uterus was completely evacuated. For this reason it became the practice to curette the vault and four quadrants of the uterus with a small Friedman curette after use of the Karman catheter. Small polyp forceps proved to be of little value for detecting adherent fragments of placenta. In addition, scrupulous attention was paid to antisepsis, povidone-iodine being liberally applied to the vagina before and after the catheter had been used.
The improvement in technique that occurred as the study progressed is reflected in the results for groups 2 and 3 when compared with those for group 1 . There was a sharp fall in blood loss and suction time. In the last 45 patients only two were admitted to hospital, both requiring only observation, and this improvement has been maintained subsequently. Nevertheless, we continue to impress on patients how essential it is for them to contact the hospital if they develop any symptoms after the termination. The real anxiety about terminations, particularly in young women, is the possible complication of pelvic sepsis with the aftermath of infertility. No figures are available on how widespread this problem is, but in the future serious consideration may have to be given to its prevention.

There was a sharp reduction in the number of patients who complained of any discomfort during the procedure once some form of local anaesthetic was employed. Penfield ${ }^{8}$ reported the results of 600 first-trimester outpatient abortions and considers that paracervical block relaxes the cervix. Both paracervical and intracervical block eliminated the discomfort of passing the catheter through the cervix, but some uterine discomfort, usually a persistent dull ache, remained while suction was applied. It is in this latter respect that the intracervical block has been noticeably more effective as an analgesic than the paracervical block. The improvement in pain response coincided with a general improvement in technique, so it is not possible to be certain whether it can all be ascribed to the local anaesthetic.

The time taken to evacuate the uterus is particularly important in a patient who is fully conscious throughout the procedure. The mean time of 3 minutes 28 seconds in group 1 is not excessive but undoubtedly rapid evacuation by suction leaves more time to ensure that the uterus is empty. The reduction in suction time in groups 2 and 3 was partly due to greater experience with the technique but was also assisted by increasing the size of the rear eye of the catheter. Suction time has been further reduced by between 30 and 90 seconds by the use of a modification of the Malmström vacuum extractor ${ }^{8}$ in place of the standard electric pump. Recently it has been found that an $8-\mathrm{mm}$ catheter is more effective than the $6-\mathrm{mm}$ catheter for evacuating the uterus when the pregnancy is of more than eight weeks' duration.

Undoubtedly the good relationship established between the patient and members of the staff was a major factor responsible for the acceptance by the patient of the procedure without a general anaesthetic. The interview with the social worker after the patient had been accepted for termination was particularly valuable. Usually the patient was so agitated at the first interview with the doctor that she remembered little of what she was told. By the time she was seen by the social worker, however, she often had many questions about the procedure. Experience obtained from the few patients who missed this interview has shown that they are so anxious by the time they reach the operating theatre that the procedure is frightening for them and becomes technically much more difficult to carry out.

The good rapport achieved with the patient proved valuable for the satisfactory attendance at the subsequent follow-up clinics. An $87 \%$ follow-up at six weeks ensured that these women, who are a high-risk group so far as future unplanned pregnancies are concerned, all received contraceptive advice. This result suggests that legal abortion may be an important adjunct to a comprehensive family planning service. Subsequent follow-up, which will be reported later, will show how many of these women used the contraception prescribed and how successful they were in avoiding pregnancies. One of the problems already encountered has been that $10 \%$ of the patients have given false addresses or have moved out of the area.

The advantages to the patient of the Karman catheter technique are considerable. The catheter has a small diameter so that dilatation of the cervix with the possible complication of cervical incompetence in a subsequent pregnancy does not exist. Primary haemorrhage does not occur and the risk of secondary haemorrhage is slight. The procedure is rapid and 
relatively painless, thus obviating the need for a general anaesthetic and, in most cases, for any form of premedication. In consequence the procedure can be organized on an outpatient basis and the patient is not required to starve before coming to hospital. If she is working she need take only the day off, or if she has children their care while she is in hospital does not present the same problem as does an admission of 48-72 hours, which is required in many hospitals for a routine vaginal termination of pregnancy. It could be argued that this makes the whole process too easy for the patient, encouraging a careless attitude towards the need for contraception in the future. This has not been our experience to date. During the eight months the method has been used by us 250 outpatient terminations have been performed. With few exceptions we have been impressed by the fact that it is the dislike of having to request and then to go through with an abortion that is the major deterrent rather than whether or not the termination procedure itself is unpleasant. To some extent the fact that the patient is awake during the procedure seems to increase her sense of involvement and consequently the responsibility of her decision.

Our experience suggests that if it remains the policy for large numbers of legal abortions to be performed in National Health Service hospitals, then a small ward with an operating theatre similar to the one we have described is ideal for the purpose. The unit can also be used for most forms of minor gynaecological surgery. ${ }^{10}$ It has been reported that abortion clinics can operate successfully geographically separated from a hospital. ${ }^{6}$
Our experience is insufficient for us to comment except to say that the advantages in terms of the ready availability of expertise and facilities of incorporating such a unit in a general hospital are considerable.

We would like to thank Professor S. G. Clayton and all the consultant gynaecologists at King's College and Dulwich Hospitals for their encouragement and for allowing patients under their care to enter the study, and also Dr. F. E. Dische and the staff of the pathology and bacteriology departments at Dulwich Hospital for their contribution to the study. We are particularly indebted to Sister Toone and all our ward staff at Dulwich Hospital for their unfailing support and kindness to all the patients. We would like to thank Miss Cynthia Knight for preparing the tables and figures. Two of us (S.C.L. and S.L.) are in tenure of grants from the Simon Population Trust.

\author{
References \\ 1 On the State of the Public Health, London, H.M.S.O., 1971. \\ 2 Beard, R. W., Abortion and Hospitals, Conference on Legal Abortion in \\ England, Amsterdam, 1970. \\ ${ }^{3}$ Keith, S., Health Trends, 1971, 3, 52. \\ 4 Karman, H., Report to International Planned Parenthood Federation, \\ October 1970 \\ ${ }^{5}$ Strausz, I. K., Schulman, H., Obstetrics and Gynecology, 1971, 38, 199. \\ 6 Potts, M., and Branch, B. N. Lancet, 1971, 2, 651. \\ 7 Beric, B. M and Kupresamin, M., Lancet, 1971, 2, 619 \\ 8 Penfield. A. J., New York State Fournal of Medicine, 1971, 71, 1185. \\ 9 Penfield, A. J., New York State fournal of Medicine, \\ 10 Clayton, S. G., et al., Lancet, 1971, 2, 1197.
}

\title{
Diagnosis of Tuberculosis in a General Hospital
}

\author{
B. H. R. STACK
}

British Medical fournal, 1971, 4, 610-612

\section{Summary}

In a survey of 71 new cases of tuberculosis diagnosed in a general hospital the average interval between admission and diagnosis of tuberculosis (the diagnostic interval) ranged between 10 days for intrathoracic tuberculosis and 20 days for genitourinary tuberculosis. The average diagnostic interval was $\mathbf{1 0 . 9}$ days when tuberculosis was included in the initial differential diagnosis, and 22.8 days when other diagnoses were made. Undue delay in diagnosis occurred in 17 patients $(24 \%)$. In eight this was due to failure to include tuberculosis in the initial differential diagnosis. Earlier diagnosis might have saved three of the five patients who died.

In 21 patients $(30 \%)$ a history of predisposing factors or associated illness was obtained. Ten of these had suffered from previous tuberculosis.

Western General Hospital, Edinburgh 4

B. H. R. STACK, M.B., CH.B., M.R.C.P.(LOND.,ED.), Senior Medical Registrar (Present address: Chest Unit, City Hospital, Edinburgh EH10 5SB)
The vital factor in diagnosis of tuberculosis in general hospital patients is consideration of this condition in the diagnosis of any unexplained illness, especially where a history of previous tuberculosis or a recognized predisposing factor is obtained.

\section{Introduction}

Though the notification rate for new cases of tuberculosis has fallen sharply over the past 13 years the average number of new cases notified annually in Edinburgh (population about 500,000 ) for the years 1964 to 1968 inclusive was 246. Twenty-one per cent. of these were diagnosed in general hospitals. Such patients differ from those presenting for the first time at chest clinics in that the clinical features are often atypical and a higher proportion suffer from non-respiratory tuberculosis.

In general hospitals the junior medical staff are usually less alert to the possibility of tuberculosis in a diagnostic problem than those who work in specialized respiratory units. They have less experience of the appropriate investigations to be carried out and in the interpretation of the results. It might thus be expected that long delay in the diagnosis of tuberculosis would occur in some cases. For this reason a survey of all new cases of tuberculosis diagnosed in this hospital in the years 1963 to 1968 inclusive was carried out and the results are reported here. 